\title{
Statistics of finite-time Lyapunov exponents in a random time-dependent potential
}

\author{
H. Schomerus* and M. Titov ${ }^{\dagger}$ \\ Max-Planck-Institut für Physik Komplexer Systeme, Nöthnitzer Strasse 38, 01187 Dresden, Germany
}

(Received 17 April 2002; published 12 December 2002)

\begin{abstract}
The sensitivity of trajectories over finite-time intervals $t$ to perturbations of the initial conditions can be associated with a finite-time Lyapunov exponent $\lambda$, obtained from the elements $M_{i j}$ of the stability matrix $M$. For globally chaotic dynamics, $\lambda$ tends to a unique value (the usual Lyapunov exponent $\lambda_{\infty}$ ) for almost all trajectories as $t$ is sent to infinity, but for finite $t$ it depends on the initial conditions of the trajectory and can be considered as a statistical quantity. We compute for a particle moving in a randomly time-dependent, one-dimensional potential how the distribution function $P(\lambda ; t)$ approaches the limiting distribution $P(\lambda ; \infty)$ $=\delta\left(\lambda-\lambda_{\infty}\right)$. Our method also applies to the tail of the distribution, which determines the growth rates of moments of $M_{i j}$. The results are also applicable to the problem of wave-function localization in a disordered one-dimensional potential.
\end{abstract}

DOI: 10.1103/PhysRevE.66.066207

PACS number(s): 05.45.-a, 05.40.-a, 42.25.Dd, 72.15.Rn

\section{INTRODUCTION}

In this work, we give a uniform description of the complete asymptotic statistics of the finite-time Lyapunov exponent for a particle moving in a randomly time-dependent, one-dimensional potential. The Lyapunov exponent $\lambda_{\infty}$ characterizes the sensitivity of trajectories to small perturbations of the initial conditions and plays a fundamental role in the characterization of systems which display deterministic chaos [1]. The Lyapunov exponent is defined in the joint limits of vanishing initial perturbation and infinitely large times. In a hyperbolic Hamiltonian system, $\lambda_{\infty}$ may be obtained from any nonperiodic trajectory, because for arbitrarily long times the trajectories uniformly explore the complete phase space.

A widely studied generalization of $\lambda_{\infty}$ is the finite-time Lyapunov exponent [1-17], which is defined for finite stretches (time interval $t$ ) of trajectories (generalizations to finite perturbations also exist [18]). The sensitivity of the dynamics to initial perturbations is given by the stability matrix map $M$, which is the linearization of the map of initial coordinates to final coordinates. In terms of elements $M_{i j}$ of $M$, the finite-time Lyapunov exponent may then be defined as

$$
\lambda=\frac{1}{2 t} \ln M_{i j}^{2} .
$$

In contrast to $\lambda_{\infty}, \lambda$ is not a unique number independent of the initial conditions, but a fluctuating quantity with a distribution function $P(\lambda ; t)$ (defined by uniformly sampling all initial conditions in phase space). This distribution function determines, e.g., the generalized entropy and dimension spectra of dynamical systems [1], and more practically the weak-localization correction to the conductance [19] and the shot-noise suppression [20,21] in mesoscopic systems. Finite-time Lyapunov exponents also determine the wavefront stability of acoustic and electromagnetic wave propa-

\footnotetext{
*Electronic address: henning @mpipks-dresden.mpg.de

†Electronic address: titov@mpipks-dresden.mpg.de
}

gation through a random medium, in the ray-acoustics/rayoptics regime of short wavelengths (for a recent application, see Refs. [22,23]). Moreover, they have shifted into the focus of attention due to recent advances in the understanding of the role of the Lyapunov exponents for quantum-chaotic wave propagation [24-30]: It has been observed that under certain conditions the Lyapunov exponent can be extracted from the decay of the overlap of two wave functions which are propagated by two slightly different Hamiltonians (the so-called Loschmidt echo). Since the overlap is studied as a function of time, the distribution of the finite-time Lyapunov exponent is directly relevant for these investigations. This extends also to related semiclassical time scales, such as the Ehrenfest time $\sim(\ln \hbar) / \lambda$, which is a semiclassical estimate of the diffraction time of wave packets due to the chaotic classical dynamics.

In the limit of infinite time $t$, the distribution function $P(\lambda ; t)$ in a completely chaotic phase space tends to the limiting form $P(\lambda ; \infty)=\delta\left(\lambda-\lambda_{\infty}\right)$. For large but finite $t$, the bulk of the distribution function can be approximated by a Gaussian centered around $\lambda_{\infty}$, with the width vanishing $\propto t^{-1 / 2}$ as $t \rightarrow \infty$. However, many of the properties determined by $P(\lambda ; t)$ (like the generalized entropy and dimension spectra) cannot be calculated from the Gaussian bulk of the distribution function [1].

In this paper, we investigate for a particle moving in a one-dimensional randomly time-dependent potential with Gaussian statistics how $P(\lambda ; t)$ approaches the limiting distribution function $P(\lambda ; \infty)=\delta\left(\lambda-\lambda_{\infty}\right)$ for large times. Our approach uniformly applies both to the bulk as well as to the far tail $\lambda \gg \lambda_{\infty}$ of the distribution function. We find that the cumulant-generating function of $P(\lambda ; t)$,

$$
\eta(\xi)=\ln \langle\exp (\xi t \lambda)\rangle=\sum_{n=1}^{\infty}\left\langle\left\langle\lambda^{n}\right\rangle\right\rangle \frac{(\xi t)^{n}}{n !}
$$

(where the average $\langle\cdot\rangle$ is over realizations of the fluctuating potential and $\langle\langle\cdot\rangle\rangle$ denotes the cumulants), takes the asymptotic form

$$
\eta(\xi)=\mu(\xi) t / t_{c}+O\left(t^{0}\right),
$$


with $\mu(\xi)$ a universal function (within the statistical model) and

$$
t_{c}=\lambda_{\infty}^{-1} \mu^{(1)}
$$

a system-specific time scale which can be determined from the infinite-time-Lyapunov exponent and the constant $\mu^{(1)}$ $=d \mu /\left.d \xi\right|_{\xi=0}$ (by definition, $d \eta /\left.d \xi\right|_{\xi=0}=\lambda_{\infty} t$ ). The function $\mu(\xi)$ is given by the leading eigenvalue of a second-order differential equation in which $\xi$ appears as a parameter. This eigenvalue can be calculated perturbatively in $\xi$, which generates the cumulants of $\lambda$. The values of $\mu$ at integer $\xi$ determine the asymptotic growth rates $(1 / t) \ln \left\langle M_{i j}^{\xi}\right\rangle$ $=\operatorname{Re} \mu(\xi) / t_{c}$ of moments of stability-matrix elements. For the positive moments $(\xi>0)$, we find that these values are given by the leading eigenvalue of finite-dimensional matrices.

In practical applications, an ensemble of independent trajectories through a randomly fluctuating potential can be realized in different ways. If the potential also has a random spatial dependence with a short correlation length (on top of the random temporal dependence), independent trajectories can be realized by choosing different initial conditions in phase space (most appropriately, distributed with the canonically invariant uniform phase-space density). It should also be noted that a random time-dependent potential is often considered as a statistical model for the ergodic properties of hyperbolic chaotic motion, in the spirit of the early work of Chirikov [31]. The time dependence of the potential then mimics the dependence of the potential in the eigentime along the trajectory. In the context of finite-time Lyapunov exponents, there have been indications that a statistical description is usually valid for the chaotic background of its distribution [16], while system-specific deviations may exist in some exceptional cases even in the bulk of the distribution function [15]. Moreover, these findings indicate that the assumption of Gaussian statistics of the fluctuations is not restrictive. While the statistical model considered in this work is tailored to a specific class of Hamiltonian systems, it can be modified straightforwardly to other classes of chaotic systems (this is briefly described at the end of this paper).

The problem of finite-time Lyapunov exponents in the random time-dependent potential is equivalent to the problem of wave localization in a random one-dimensional potential [32-36], because the equations of motion for the matrix elements $M_{i j}$ are formally equivalent to the corresponding Schrödinger equation [10,37]. Indeed, the Fokker-Planck equation employed in this work is based on the phase formalism described, e.g., in Refs. [38-40]. Hence, the asymptotic statistics of the finite-time Lyapunov exponent presented in this work is of direct interest and can be transferred to this field of research [41]. A number of additional areas of application of our method come into scope if one considers the vast arena of problems which can be analyzed by products of random matrices, since the finitetime Lyapunov exponents are a valuable way to characterize the eigenvalues of these products [10].

The plan of this paper is as follows. In Sec. II, we formulate the problem of finite-time Lyapunov exponents in the one-dimensional random time-dependent potential. In Sec. III, we show how the cumulant-generating function can be related to the parametrized eigenvalue of a second-order differential equation, and that the cumulants can be calculated systematically. Moments of $M_{i j}$ are calculated in Sec. IV. We close the paper with a discussion and conclusions in Sec. V.

\section{FORMULATION OF THE PROBLEM}

\section{A. Statistical model}

Let us consider an ensemble of time-dependent Hamiltonian system with one degree of freedom (canonically conjugated coordinates $x, p$ ), and the Hamiltonian given by

$$
H=\frac{p^{2}}{2 m}+V(x, t) \text {. }
$$

Here $V(x, t)$ is a randomly time-dependent potential and $m$ is the mass. Shortly we will see that the stability of trajectories obtained from Hamiltonian (5) is determined by properties of the curvature of the potential. We allow for a timeindependent mean curvature

$$
\left\langle\frac{\partial^{2} V}{\partial x^{2}}\right\rangle=V_{2}
$$

which may arise from a static background potential, while temporal fluctuations of the curvature are assumed to be Gaussian and $\delta$-function-correlated,

$$
\left\langle\frac{\partial^{2} V\left(x, t_{1}\right)}{\partial x^{2}} \frac{\partial^{2} V\left(x, t_{2}\right)}{\partial x^{2}}\right\rangle-V_{2}^{2}=2 D \delta\left(t_{1}-t_{2}\right) .
$$

The constant $D$ is similar to a diffusion constant, but not identical with conventional diffusion constants of motion in phase space. [In the specific model of Refs. [22,23], $D$ can be related to the temporal and spatial fluctuation properties of the potential $V(x, t)$.]

Equation (5) along with Hamilton's equations of motion and Eqs. (6) along with the assumption of Gaussian fluctuations completely specify the statistical model of Hamiltonian dynamics which we investigate in this paper. As already mentioned in the Introduction, statistical models of this kind have a long history in the description of dynamical systems, not only for truly time-dependent cases but also as an approximation of the pseudorandomness induced by chaotic dynamics, with the underlying assumption that different stretches of typical trajectories are practically uncorrelated. Although we restrict our mathematical analysis to the statistical model with Gaussian fluctuations, the general findings should also carry over to other random potentials, as long as the correlations are short-ranged and the variance of the random values is finite. This expectation is supported by the certain degree of universality found in chaotic dynamical systems, as well as by the indications of universal wavefunction statistics in one-dimensional localization. For detailed investigations of the applicability of statistical approaches to these models, refer to the works mentioned in the Introduction. 
In order to investigate the stability properties of trajectories, we introduce the map $\mathcal{F}_{t}$ which propagates initial conditions $\left(x_{i}, p_{i}\right)$ over a time interval $t$ to the final coordinates $\left(x_{f}, p_{f}\right)=\mathcal{F}_{t}\left(x_{i}, p_{i}\right)$. The stability matrix $M$ is the linearization of the map $\mathcal{F}_{t}$ and describes the sensitivity of the final coordinates to a small perturbation of the initial conditions,

$$
M=\frac{\partial\left(x_{f}, p_{f}\right)}{\partial\left(x_{i}, p_{i}\right)}=\left(\begin{array}{ll}
M_{11} & M_{12} \\
M_{21} & M_{22}
\end{array}\right) .
$$

Area preservation of the dynamics in phase space entails the property $\operatorname{det} M=1$ of the stability matrix.

We are interested in the evolution of the stability matrix with given initial conditions and increasing time interval $t$. According to Hamilton's equations of motion, the stability matrix fulfills the differential equation

$$
\frac{d M}{d t}=K M, \quad K=\left(\begin{array}{cc}
0 & m^{-1} \\
v & 0
\end{array}\right),
$$

where the function $v(t)$ in the matrix $K$ is given by

$$
v(t)=-\left.\frac{d^{2} V}{d x^{2}}\right|_{x=x_{f}(t)} .
$$

Differential equation (8) is supplemented by the initial conditions

$$
M(0)=\operatorname{diag}(1,1),
$$

corresponding to the identification of the initial and final coordinate systems for $t=0$. The statistical properties of $v$ directly follow from Eqs. (6),

$$
\langle v(t)\rangle=-V_{2}, \quad\left\langle v\left(t_{1}\right) v\left(t_{2}\right)\right\rangle-V_{2}^{2}=2 D \delta\left(t_{1}-t_{2}\right) .
$$

Both $D$ as well as the mass $m$ can be eliminated from the subsequent analysis by rescaling quantities in the following way:

$$
\begin{gathered}
t=t_{c} t^{\prime}, \quad v=(D / m) t_{c} v^{\prime}, \quad V_{2}=(D / m) t_{c} V_{2}^{\prime}, \\
M_{12}=\left(t_{c} / m\right) M_{12}^{\prime}, \quad M_{21}=\left(m / t_{c}\right) M_{21}^{\prime}, \\
M_{11}=M_{11}^{\prime}, \quad M_{22}=M_{22}^{\prime} .
\end{gathered}
$$

Here we defined the characteristic time scale

$$
t_{c}=m^{2 / 3} D^{-1 / 3} \text {. }
$$

[In the course of our analysis, we will see that this time scale also can be found from Eq. (4).] The rescaled (primed) quantities fulfill Eqs. (8),(10),(11), with $D=m=1$. Also note that the rescaling leaves the property $\operatorname{det} M=1$ invariant.

\section{B. Relation to one-dimensional localization}

The set of linear first-order differential equations (8) can be decoupled by converting them into second-order differential equations. It is useful to note (as mentioned in the Intro- duction) that the equations for the elements $M_{11}$ and $M_{12}$ are equivalent to the Schrödinger equation, at energy $E$ $=V_{2} / m$, of a particle of mass $\hbar^{2} / 2$ in a one-dimensional random potential $\left(v+V_{2}\right) / m$ (of vanishing mean), with $t$ playing the role of the spatial coordinate,

$$
\frac{d^{2} M_{11}}{d t^{2}}=\frac{v}{m} M_{11}, \quad \frac{d^{2} M_{12}}{d t^{2}}=\frac{v}{m} M_{12},
$$

while the other matrix elements are directly related to them by

$$
M_{21}=m \frac{d M_{11}}{d t}, \quad M_{22}=m \frac{d M_{12}}{d t} .
$$

The problem of finite-time Lyapunov exponents hence is closely related to the problem of one-dimensional localization in a random potential, in which the Lyapunov exponent corresponds to the inverse decay length of the wave function.

\section{CUMULANTS OF THE FINITE-TIME LYAPUNOV EXPONENT}

We now solve the problem of finding the probability distribution function of matrix elements $M_{i j}$ within the statistical model of chaotic dynamics, defined by the evolution equation (8) for $M$, with initial condition (10), and the statistical properties (11) of the random function $v$. For the sake of definiteness we will consider in this section the statistics of the upper diagonal element $M_{11}$. The results directly carry over to the other elements of $M$, as is discussed in Sec. IV C.

\section{A. Cumulant-generating function as an eigenvalue}

We introduce the quantities

$$
u=\frac{1}{2} \ln M_{11}^{\prime 2}, \quad z=\frac{M_{21}^{\prime}}{M_{11}^{\prime}},
$$

where the relation $u=\lambda t$ to the finite-time Lyapunov exponent $\lambda$ is established by Eq. (1) [note that $M_{11}=M_{11}^{\prime}$ in the rescaling Eq. (12)]. According to Eqs. (8) and (12), $u$ and $z$ fulfill the differential equations

$$
\frac{d u}{d t^{\prime}}=z, \quad \frac{d z}{d t^{\prime}}=v^{\prime}-z^{2} .
$$

Note that the evolution equation of $z$ decouples from $u$ and can be interpreted as a Langevin equation. Hence the distribution $P\left(z ; t^{\prime}\right)$ can be calculated from a Fokker-Planck equation, which was considered before in the context of wave-function localization $[39,40]$,

$$
\begin{gathered}
\partial_{t^{\prime}} P\left(z ; t^{\prime}\right)=\mathcal{L}_{z} P\left(z ; t^{\prime}\right), \\
\mathcal{L}_{z} \cdot=\partial_{z}\left(z^{2}+V_{2}^{\prime}+\partial_{z}\right) .
\end{gathered}
$$

For large $t^{\prime}$, the distribution function $P\left(z ; t^{\prime}\right)$ approaches the stationary solution $[38-40]$ 


$$
\begin{gathered}
P_{\text {stat }}(z)=\widetilde{N} \int_{-\infty}^{z} d y K(y, z), \\
K(y, z)=e^{\left(y^{3}-z^{3}\right) / 3+V_{2}^{\prime}(y-z)}, \\
\widetilde{N}=\pi^{-2}\left[\mathrm{Ai}^{2}\left(-V_{2}^{\prime}\right)+\mathrm{Bi}^{2}\left(-V_{2}^{\prime}\right)\right]^{-1} .
\end{gathered}
$$

Here $\mathrm{Ai}$ and $\mathrm{Bi}$ are Airy functions. The normalization constant is directly related to the integrated density of states in the localization problem $[38-40]$. For $V_{2}^{\prime}=0, \tilde{N}$ $=3^{5 / 6} 2^{-1 / 3} \pi^{-1 / 2} /[\Gamma(1 / 6)]$. Because $d u / d t=z / t_{c}$, it is clear [40] that the infinite-time Lyapunov exponent can be obtained from $\lambda_{\infty}=\langle z\rangle / t_{c}$; this relation will be demonstrated explicitly in Sec. III C.

The Fokker-Planck equation for the joint distribution function $P\left(u, z ; t^{\prime}\right)$ is given by

$$
\partial_{t^{\prime}} P=-z \partial_{u} P+\mathcal{L}_{z} P .
$$

This Fokker-Planck equation with $V_{2}^{\prime}=0$ has been derived in Ref. [19] for the autonomous chaotic scattering of a particle from a dilute collection of scatterers (with more than one degree of freedom).

The joint distribution function $P\left(u, z ; t^{\prime}\right)$ does not approach a stationary limit because $u$ runs away to infinitely large values. In order to analyze the behavior of the distribution function $P\left(u, z ; t^{\prime}\right)$ for large times, we convert the Fokker-Planck equation (20) into an eigenvalue problem which discriminates between the different time scales involved in this evolution. For this purpose, we introduce into Eq. (20) the ansatz

$$
P\left(u, z ; t^{\prime}\right)=\int_{-i \infty}^{+i \infty} \frac{d \xi}{2 \pi i} \sum_{n=0}^{\infty} \exp \left[\mu_{n}(\xi) t^{\prime}-\xi u\right] f_{n}(\xi, z) .
$$

(The integration contour along the imaginary axis corresponds to a Fourier transformation.) It follows that the functions $f_{n}$ fulfill the differential equation

$$
\mu_{n} f_{n}(\xi, z)=\left(\xi z+\mathcal{L}_{z}\right) f_{n}(\xi, z)
$$

in which $\mu_{n}$ and $\xi$ appear as parameters. However, in order to obtain a meaningful probability distribution function (21), we have to impose boundary conditions on $f_{n}(\xi, z)$ at $z \rightarrow$ $\pm \infty$. It is convenient to express these boundary conditions by the requirement

$$
\mathcal{P} \int_{-\infty}^{\infty} d z f_{n}(\xi, z) z<\infty
$$

Here $\mathcal{P}$ denotes the principal value with respect to the integration boundaries at $\pm \infty$. Condition (22b) follows from the behavior $z \approx\left(t^{\prime}-t_{\infty}^{\prime}\right)^{-1}$ of the solution of the differential equation (17) close to times $t^{\prime} \approx t_{\infty}^{\prime}$, where $|z| \rightarrow \infty$ (and hence $v^{\prime}$ can be ignored). In practical terms, the condition (22b) guarantees that the drift of $u$ remains finite for all times.
Equations (22) form an eigenvalue problem, since condition (22b) only can be fulfilled for a discrete set of numbers $\mu_{n}$-note that these eigenvalues depend on the parameter $\xi$. At $\xi=0$, there is a finite gap between the largest eigenvalue $\mu_{0}$ and the second-largest eigenvalue $\mu_{1}$, and by continuity it is guaranteed that this gap also persists in a finite neighborhood of $\xi=0$. In the limit of large $t^{\prime}$, only the largest eigenvalue is relevant, because the other eigenvalues give rise to exponentially smaller contributions. The largest eigenvalue vanishes as $\xi \rightarrow 0$, i.e., $\mu_{0}(0)=0$, because the stationary distribution of $z$, Eq. (19), must be recovered for large times from Eq. (21) by integrating out $u$. In the following, we denote for simplicity $\mu_{0}(\xi) \equiv \mu(\xi)$.

The moments of $u$ are given by

$$
\begin{aligned}
\left\langle u^{n}\right\rangle & =\int_{-i \infty}^{+i \infty} \frac{d \xi}{2 \pi i} \int_{-\infty}^{\infty} d u \exp \left(\mu t^{\prime}-\xi u\right) f(\xi) u^{n} \\
& =\lim _{\xi \rightarrow 0} \partial_{\xi}^{n} \exp \left(\mu(\xi) t^{\prime}\right) f(\xi),
\end{aligned}
$$

where the coefficients $f(\xi)=\int_{-\infty}^{\infty} d z f_{0}(\xi, z)$ are determined, in principle, by the initial condition for $P\left(u, z ; t^{\prime}\right)$ at $t^{\prime}=0$. From Eq. (23), we obtain the moment-generating function

$$
\chi(\xi)=\langle\exp (\xi u)\rangle=\exp \left(\mu(\xi) t / t_{c}\right) f(\xi),
$$

where we reintroduced the original time variable $t=t_{c} t^{\prime}$ by Eq. (12). The cumulant-generating function (2) hence takes the form of Eq. (3), including the corrections of order $t^{0}$,

$$
\eta(\xi)=\ln \chi(\xi)=\mu(\xi) t / t_{c}+\ln f(\xi) .
$$

The cumulants $\left\langle\left\langle\lambda^{n}\right\rangle\right\rangle$ of the finite-time Lyapunov exponent are obtained by expanding the generating function $\eta$ in powers of $\xi$, see Eq. (2). In terms of the coefficients of the Taylor expansion,

$$
\mu(\xi)=\sum_{n=1}^{\infty} \xi^{n} \mu^{(n)}
$$

[which starts with the linear term in $\xi$ because $\mu(0)=0$ ], according to Eqs. (2) and (25) the $n$th cumulant of $\lambda$ is then given by

$$
\left\langle\left\langle\lambda^{n}\right\rangle\right\rangle=n ! \mu^{(n)} t_{c}^{-1} t^{1-n}+O\left(t^{-n}\right) .
$$

This equation means that within the statistical model, the cumulants are universal quantities in the leading order in $t$, in the sense that the initial conditions $P(z, u ; 0)$ only enter the next-order corrections. The only system-specific parameters which enter the cumulants are the time scale $t_{c}$ and the (rescaled) strength $V_{2}^{\prime}$ of the static potential. Note that ratios of cumulants are even independent of the time scale $t_{c}$ (and hence of the parameters $D$ and $m$ of the statistical model).

The form (4) of $t_{c}$ follows from Eq. (27) when $t_{c}$ is expressed in terms of the infinite-time Lyapunov exponent $\lambda_{\infty}$ with help of the definition

$$
\lambda_{\infty}=\lim _{t \rightarrow \infty}\langle\lambda\rangle=\mu^{(1)} / t_{c} .
$$


In terms of the bare quantities of the statistical model,

$$
\lambda_{\infty}=\mu^{(1)} D^{1 / 3} m^{-2 / 3} .
$$

In the next two sections, we obtain general expressions for the expansion coefficients $\mu^{(n)}$ and calculate explicitly the proportionality factor $\mu^{(1)}=d \mu /\left.d \xi\right|_{\xi=0}$ in Eq. (29), as well as the first few coefficients $\mu^{(2)}, \mu^{(3)}, \ldots$, which determine, respectively, the variance and the leading nonGaussian corrections (higher cumulants) of the fluctuations of the finite-time Lyapunov exponent around its limiting value $\lambda_{\infty}$.

\section{B. Recursion relations for the cumulants}

We now show how the cumulants can be calculated from Eq. (27) by recursively solving a hierarchy of equations for coefficients $\mu^{(n)}$ in the Taylor expansion of $\mu(\xi)$, Eq. (26).

In analogy to Eq. (26), let us also expand the function $f_{0}(\xi, z)$ in powers of $\xi$,

$$
f_{0}(\xi, z)=\sum_{n=0}^{\infty} \xi^{n} f_{0}^{(n)}(z)
$$

With Eqs. (26) and (30), the eigenvalue problem (22) can now be written order by order in powers of $\xi^{n}$. For $n=0$, we recover the stationary variant (18) of the Fokker-Planck equation (20),

$$
\mathcal{L}_{z} f_{0}^{(0)}(z)=0
$$

which is solved by the stationary solution $f_{0}^{(0)}(z)=P_{\text {stat }}(z)$, Eq. (19). For $n>1$, the differential equations are of the form

$$
\mathcal{L}_{z} f_{0}^{(n)}(z)=-z f_{0}^{(n-1)}(z)+\sum_{l=1}^{n} \mu^{(l)} f_{0}^{(n-l)}(z) .
$$

Let us assume that we have solved the hierarchy of equations up to order $n-1$. In the next order $n$, both the unknown quantities $f_{0}^{(n)}$ as well as $\mu^{(n)}$ appear. The unknowns can be separated by integrating the differential equation (32) over $z$ from $-\infty$ to $\infty$ : The integrated left-hand side vanishes because of condition (22b) of the eigenvalue problem. The integrated right-hand side can be rearranged to give $\mu^{(n)}$,

$$
\mu^{(n)}=\int_{-\infty}^{\infty} d z\left[z f_{0}^{(n-1)}(z)-\sum_{l=1}^{n-1} \mu^{(l)} f_{0}^{(n-l)}(z)\right],
$$

which only involves quantities up to order $n-1$. Subsequently, $\mu^{(n)}$ can be inserted into Eq. (32). The function

$$
\begin{aligned}
f_{0}^{(n)}(z)= & \int_{-\infty}^{z} d y \int_{-\infty}^{y} d x K(y, z)\left[-x f_{0}^{(n-1)}(x)\right. \\
& \left.+\sum_{l=1}^{n} \mu^{(l)} f_{0}^{(n-l)}(x)\right]
\end{aligned}
$$

[with the kernel $K(y, z)$ defined in Eq. (19)] is then obtained by solving the resulting inhomogeneous differential equation with the help of the partial solution $f_{0}^{(0)}(z)$ of its homogeneous counterpart, Eq. (31). This inhomogeneous part of the functions $f_{0}^{(n)}(z)$ is fixed by the requirement that $f_{0}^{(0)}(z)$ be normalized to 1 . Adding the homogeneous solution to $f_{0}^{(n)}(z)$ in any order gives rise to additional terms in all higher orders, but these combine in such a way that they drop out of the calculation of the coefficients $\mu^{(n)}$, which hence are uniquely determined by Eq. (33a).

The recursion relations (33) can be iterated to calculate successively all cumulants of $\lambda$.

\section{Explicit expressions and numerical values}

According to Eq. (27), the two numbers $\mu^{(1)}$ and $\mu^{(2)}$ determine mean and variance of the distribution function of $\lambda$, which then is approximated by a Gaussian. The coefficient $\mu^{(1)}$ has been obtained in Ref. [40] from the FokkerPlanck equation (18) for arbitrary $V_{2}$. For the special case $V_{2}=0$, the two coefficients $\mu^{(1)}$ and $\mu^{(2)}$ have been obtained in Ref. [19] from the Fokker-Planck equation (20). However, the deviations from the Gaussian distribution function are not at all negligible for many chaotic systems, which is most clearly displayed in their generalized dimension and entropy spectra [1]. As we have seen in Sec. III B, our approach of reduction to the eigenvalue problem (22) allows us to analyze the non-Gaussian deviations by the higher cumulants of $\lambda$. [In Sec. IV, we show that one can even obtain from our analysis the positive moments $M_{11}^{\xi}, \xi>0$, which are determined by the far tail $\lambda \gg \lambda_{\infty}$ of $P(\lambda ; t)$, while the bulk of the distribution is essentially irrelevant for these moments.]

Explicit expressions for the first few coefficients $\mu^{(1)}$, $\mu^{(2)}, \mu^{(3)}$, and $\mu^{(4)}$ result from Eq. (33a),

$$
\mu^{(1)}=\int_{-\infty}^{\infty} d z z f_{0}^{(0)}(z)
$$

$$
\begin{gathered}
\mu^{(2)}=\int_{-\infty}^{\infty} d z\left(z-\mu^{(1)}\right) f_{0}^{(1)}(z), \\
\mu^{(3)}=\int_{-\infty}^{\infty} d z\left[\left(z-\mu^{(1)}\right) f_{0}^{(2)}(z)-\mu^{(2)} f_{0}^{(1)}(z)\right], \quad(34 \mathrm{c}) \\
\mu^{(4)}=\int_{-\infty}^{\infty} d z\left[\left(z-\mu^{(1)}\right) f_{0}^{(3)}(z)-\mu^{(2)} f_{0}^{(2)}(z)-\mu^{(3)} f_{0}^{(1)}(z)\right]
\end{gathered}
$$

where $f_{0}^{(0)}(z)=P_{\text {stat }}(z)$ is given by the stationary distribution function of $z$, Eq. (19), while the other functions follow from Eq. (33b),

$$
f_{0}^{(1)}(z)=\int_{z>y>x} d y d x K(y, z)\left(\mu^{(1)}-x\right) f_{0}^{(0)}(x)
$$



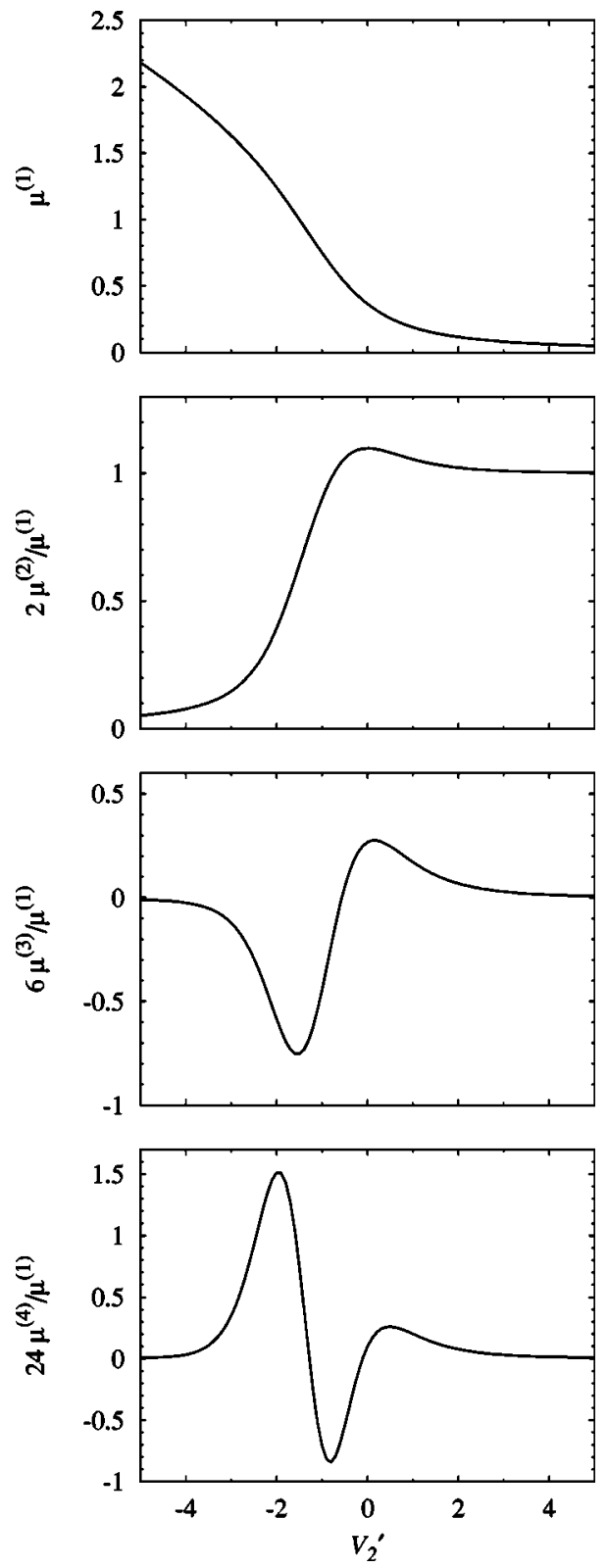

FIG. 1. Coefficient $\mu^{(1)}$ of the first cumulant, and the ratios $n ! \mu^{(n)} / \mu^{(1)}$ for the coefficients of the second, third, and fourth cumulant [cf. Eq. (27)], as a function of the strength $V_{2}^{\prime}$ of the static background potential.

$$
\begin{aligned}
f_{0}^{(2)}(z)= & \int_{z>y>x} d y d x K(y, z)\left[\left(\mu^{(1)}-x\right) f_{0}^{(1)}(x)\right. \\
& \left.-\mu^{(2)} f_{0}^{(0)}(x)\right] \\
f_{0}^{(3)}(z)= & \int_{z>y>x} d y d x K(y, z)\left[\left(\mu^{(1)}-x\right) f_{0}^{(2)}(x)\right. \\
& \left.-\mu^{(2)} f_{0}^{(1)}(x)-\mu^{(3)} f_{0}^{(0)}(x)\right] .
\end{aligned}
$$

The coefficient $\mu^{(1)}$ is then given by [40]
TABLE I. The first eight coefficients $n ! \mu^{(n)}$ of the cumulants of finite-time Lyapunov exponents [cf. Eq. (27)], in the absence of the static background potential $\left(V_{2}^{\prime}=0\right)$.

\begin{tabular}{ccccc}
\hline \hline$n$ & 1 & 2 & 3 & 4 \\
\hline$n ! \mu^{(n)}$ & 0.365 & 0.401 & 0.0975 & 0.0361 \\
$n$ & 5 & 6 & 7 & 8 \\
\hline$n ! \mu^{(n)}$ & -0.266 & -0.628 & -0.554 & 3.71 \\
\hline \hline & & & \\
& $\mu^{(1)}=\frac{1}{2} \frac{d}{d V_{2}^{\prime}} \log \widetilde{N}$, &
\end{tabular}

where $\widetilde{N}$ is given in Eq. (19), while the cumulants for $n \geqslant 2$ can be obtained quickly by numerical integration of $2 n$-fold integrals. The effort of integration can be greatly reduced down to the expense equivalent to a single integral, because the integrand factorizes. An efficient recursive scheme is described in the Appendix. In Fig. 1, we plot the coefficient $\mu^{(1)}$ and the ratios $n ! \mu^{(n)} / \mu^{(1)}$ for $n=2,3,4$ as a function of $V_{2}^{\prime}$. The non-Gaussian corrections are largest around $V_{2}^{\prime}$ $=0$, while they become irrelevant for large negative or positive values of $V_{2}^{\prime}$.

For large negative values $V_{2}^{\prime} \ll-1$, the growth rate $z$ of $u$ fluctuates only weakly around the value $z=\sqrt{-V_{2}^{\prime}}$, which in the absence of the temporal fluctuations in $v$ would be a stable equilibrium point for $z$, see Eq. (17). Hence $u$ grows linearly in time with almost negligible fluctuations.

For $V_{2}^{\prime} \gg 1$, the Lyapunov exponent becomes small because of the stabilizing influence of the confining background potential. [In the context of wave localization, this corresponds to the well-known limit of a large Fermi energy $E \sim V_{2}^{\prime}$ (cf. Sec. II B).] The matrix elements $M_{i j}$ then oscillate with an almost fixed frequency $\sim \sqrt{V_{2}^{\prime}}$, as can be seen from Eq. (8). The coefficients $n ! \mu^{(n)} / \mu^{(1)} \rightarrow \delta_{1 n}+\delta_{2 n}$, with $\delta_{m n}$ the Kronecker symbol, and the Gaussian approximation

$$
\mu_{\text {Gaussian }}(\xi)=\mu^{(1)}\left(\xi+\frac{1}{2} \xi^{2}\right)
$$

becomes valid.

Analytical results can be found in the case $V_{2}^{\prime}=0$ for the first two coefficients,

$$
\begin{gathered}
\mu^{(1)}=\frac{(3 / 2)^{1 / 3} \sqrt{\pi}}{\Gamma(1 / 6)}, \\
\mu^{(2)}=\frac{5 \pi^{2}}{18} \widetilde{N}-\frac{\pi}{2 \sqrt{3}} \widetilde{N}_{3} F_{2}\left(1,1, \frac{7}{6} ; \frac{3}{2}, \frac{3}{2} ; \frac{3}{4}\right),
\end{gathered}
$$

where $\widetilde{N}\left(V_{2}^{\prime}=0\right)=3^{5 / 6} 2^{-1 / 3} \pi^{-1 / 2} /[\Gamma(1 / 6)]$, while ${ }_{3} F_{2}$ is a generalized hypergeometric function. Incidentally, the numerical value given for $\mu^{(2)}$ in Ref. [19] is wrong, but the analytic expression given in that paper is equivalent to Eqs. (34b) and (38b). In Table I, we tabulate the numerical values of the first eight coefficients $n ! \mu^{(n)}$ for $V_{2}^{\prime}=0$. 


\section{MOMENTS OF $M_{i j}$}

\section{A. Formally exact expressions}

In view of Eqs. (16) and (24), we find that the exponential growth rates of the moments of $M_{11}$ are given by the largest eigenvalue $\mu(\xi)$ of Eq. (22) at integer values of $\xi$ [42],

$$
\frac{d \ln \left\langle M_{11}^{\xi}\right\rangle}{d t}=\operatorname{Re} \frac{\mu(\xi)}{t_{c}} .
$$

As we will now show, for positive integer values of $\xi$ the eigenvalue problem (22) can be reduced to a matrix eigenvalue problem of finite dimension. For the first few moments, the leading eigenvalue can be calculated explicitly, while for larger values it is formally given by the largest root of the corresponding characteristic polynomial.

In order to obtain a solution of the differential equation (22a), we write

$$
f_{0}(\xi, z)=\int_{-\infty}^{z} d y K(y, z) \frac{g(z)}{g(y)^{2}}
$$

[with the kernel $K(y, z)$ defined in Eq. (19)], and obtain for $g$ the differential equation

$$
(\mu-\xi z) g=-\left(z^{2}+V_{2}^{\prime}\right) \partial_{z} g+\partial_{z}^{2} g
$$

(a triconfluent Heun's equation with singularity at $1 / z=0$ ). We introduce into this equation the polynomial ansatz

$$
g(z)=\sum_{n=0}^{\xi} c_{n} z^{n}
$$

Power matching results in the following backward-recursion relation:

$$
(\xi-n) c_{n}=\mu c_{n+1}+(n+2)\left[V_{2}^{\prime} c_{n+2}-(n+3) c_{n+3}\right]
$$

for the coefficients $c_{n}$, with initial conditions

$$
c_{\xi}=1, \quad c_{\xi-1}=\mu, \quad c_{\xi-2}=\mu^{2} / 2 .
$$

For integer $\xi$, this backward-recursion relation terminates. We obtain functions $c_{0}(\mu), c_{1}(\mu)$, and $c_{2}(\mu)$ and an additional condition from the terms in Eq. (41) which are constant in $z$,

$$
p_{\xi}(\mu)=\mu c_{0}+V_{2}^{\prime} c_{1}-2 c_{2}=0
$$

where $p_{\xi}(\mu)$ is a polynomial of degree $\xi+1$. This polynomial can also be interpreted as the characteristic polynomial of the $[(\xi+1) \times(\xi+1)]$-dimensional matrix

$$
\left(\begin{array}{cccccccc}
0 & -V_{2}^{\prime} & 1 \times 2 & 0 & 0 & \cdots & \cdots & \cdots \\
\xi & 0 & -2 V_{2}^{\prime} & 2 \times 3 & 0 & \cdots & \cdots & \cdots \\
0 & \xi-1 & 0 & -3 V_{2}^{\prime} & 3 \times 4 & \ldots & \cdots & \cdots \\
0 & 0 & \xi-2 & 0 & -4 V_{2}^{\prime} & \ldots & \ldots & \ldots \\
0 & 0 & 0 & \xi-3 & 0 & \ddots & \cdots & \ldots \\
\vdots & \vdots & \vdots & \vdots & \ddots & 0 & (1-\xi) V_{2}^{\prime} & (\xi-1) \xi \\
\vdots & \vdots & \vdots & \vdots & \vdots & 2 & 0 & -\xi V_{2}^{\prime} \\
\vdots & \vdots & \vdots & \vdots & \vdots & 0 & 1 & 0
\end{array}\right)
$$

which is the matrix representation of the eigenvalue problem (22) in the space of the monomial expansion of $g(z)$.

The exponential growth rate $\mu(\xi)$ of the $\xi$ th moment is given by the $\operatorname{root}(\mathrm{s})$ of $p_{\xi}(\mu)$ with the largest real part [42]. In Sec. IV B, we will see for the examples $\xi=1,2$ that the other roots show up in the transient behavior of the moments.

For $V_{2}^{\prime}=0$, the values for the first few moments are given in Table II. Figure 2 shows the growth rates and the real part of the subleading eigenvalue for values of $\xi$ up to 80. A log-normal statistics of $M_{11}$ (corresponding to a Gaussian statistics of the finite-time Lyapunov exponents) would result in the quadratic dependence Eq. (37) of $\mu(\xi)$ on $\xi$, while the plot shows a weaker (approximately linear) dependence for large $\xi$. This results from the influence of the terms $\mu^{(n)} \xi^{n}$ for $n \geqslant 3$ in the complete Taylor expansion of $\mu$, Eq. (26).
The distance of the subleading eigenvalue to the leading eigenvalue increases with increasing $\xi$.

For finite $V_{2}^{\prime}$, we plotted the real parts of the leading and subleading growth rates [eigenvalues of the matrix (45)] for the first four moments in Fig. 3. The growth rate $\operatorname{Re} \mu(1)$

TABLE II. Exponential growth rates $\mu(\xi)$ of the first few moments $\left\langle M_{11}^{\xi}\right\rangle$ [cf. Eq. (39)], in the absence of the static background potential $\left(V_{2}^{\prime}=0\right)$.

\begin{tabular}{lcccc}
\hline \hline$\xi$ & 1 & 2 & 3 & 4 \\
$\mu$ & 0 & $2^{2 / 3}$ & $24^{1 / 3}$ & $84^{1 / 3}$ \\
\hline$\xi$ & 5 & 6 & 7 \\
$\mu$ & $2(14+3 \sqrt{19})^{1 / 3}$ & $(252+24 \sqrt{79})^{1 / 3}$ & $2(63+15 \sqrt{10})^{1 / 3}$ \\
\hline \hline
\end{tabular}




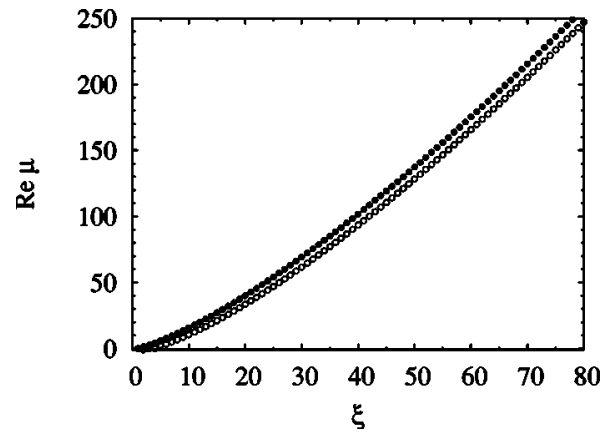

FIG. 2. Growth rates $\mu(\xi)$ of the moments $\left\langle M_{11}^{m}\right\rangle$ [cf. Eq. (39)] in the absence of the static background potential $\left(V_{2}^{\prime}=0\right)$, obtained as the largest eigenvalue of the matrix (45) (full circles). Also shown is the real part of the subleading eigenvalue of this matrix (open circles).

$=\left|\operatorname{Im} \sqrt{V_{2}^{\prime}}\right|$ of the first moment $\left\langle M_{11}\right\rangle$ follows from the roots of $p_{1}(\mu)=\mu^{2}+V_{2}^{\prime}$. Consequently, the growth rate of $\left\langle M_{11}\right\rangle$ vanishes in the case of confinement, $V_{2}^{\prime}>0$. This will be confirmed by the direct computation in Sec. IV B.

\section{B. Direct computation of the first and second moment}

In order to illustrate our findings for the growth rates of the moments $\left\langle M_{11}^{\xi}\right\rangle$, we compare the results for $\xi=1$ and $\xi=2$ to the exact results for all times (including the transient behavior). A formal solution of the differential equation (14) in terms of a series in the disorder potential is obtained by integrating Eq. (14) iteratively, under observation of the initial conditions $M_{11}=1, d M_{11} / d t=0$ for $t=0$. This gives

$$
M_{11}\left(t_{0}\right)=1+\sum_{n=1}^{\infty} \prod_{k=1}^{n} \int_{0}^{t_{k-1}} d t_{k}\left(t_{k-1}-t_{k}\right) \frac{v\left(t_{k}\right)}{m},
$$

where we introduced $t_{0}=t$ for notational convenience.

For the first moment, we can average Eq. (46) directly with help of Eq. (11),

$$
\left\langle M_{11}\right\rangle=\cos \left[\left(t / t_{c}\right) \sqrt{V_{2}^{\prime}}\right]=\frac{1}{2} e^{\left(t / t_{c}\right)} \sqrt{-V_{2}^{\prime}}+\frac{1}{2} e^{-\left(t / t_{c}\right)} \sqrt{-V_{2}^{\prime}} .
$$

For $V_{2}^{\prime}=0$, the first moment is constant and given by its initial value, $\left\langle M_{11}\right\rangle=1$. This means that negative deviations $M_{11} \ll 0$, corresponding to inverse hyperbolic motion, cancel precisely the positive deviations $M_{11} \gg 0$ of hyperbolic motion. For negative $V_{2}^{\prime}$ the first moment grows, while for positive $V_{2}^{\prime}$ it oscillates and stays of order unity. In the decomposition of the cosine into the two exponentials, we identify in the exponents the two roots $\pm \sqrt{-V_{2}^{\prime}}$ of $p_{1}(\mu)=\mu^{2}$ $+V_{2}^{\prime}$. For negative $V_{2}^{\prime}$, the subleading root hence governs the transient behavior of the first moment.

For the second moment, let us restrict ourselves for simplicity to the case $V_{2}^{\prime}=0$. We group the functions $v$ in the two factors of $M_{11}$ in pairs and then invoke the $\delta$-function correlations of Eq. (11). Performing the time-ordered integrals, we obtain
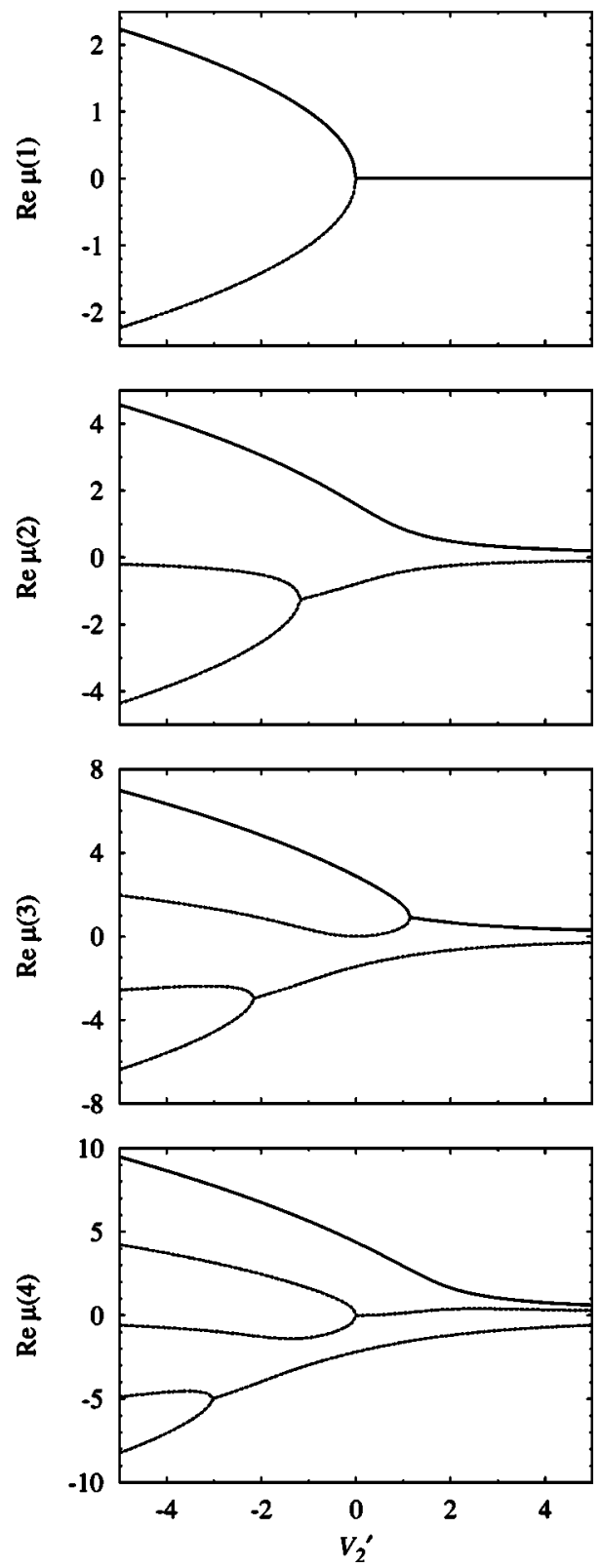

FIG. 3. Growth rates $\operatorname{Re} \mu(\xi)$ of the moments $\left\langle M_{11}^{m}\right\rangle$ [cf. Eq. (39)], for $m=1,2,3,4$, as a function of the strength $V_{2}^{\prime}$ of the static background potential. Also shown (dashed lines) are the subleading growth rates [real parts of the subleading eigenvalues of the matrix (45)].

$$
\begin{aligned}
\left\langle M_{11}^{2}\right\rangle & =1+\sum_{n=1}^{\infty}\left(2 / t_{c}^{3}\right)^{n} \prod_{k=1}^{n} \int_{0}^{t_{k-1}} d t_{k}\left(t_{k-1}-t_{k}\right)^{2} \\
& =\frac{1}{3}\left[e^{\mu(2) t / t_{c}}+e^{-(-1)^{1 / 3} \mu(2) t / t_{c}}+e^{-(-1)^{-1 / 3} \mu(2) t / t_{c}}\right] .
\end{aligned}
$$

The asymptotic growth rate of the second moment is given by the leading root $\mu(2)=2^{2 / 3}$ of the characteristic polynomial $p_{2}(\mu)=\frac{1}{2} \mu^{3}-2$, in accordance with Table II. The second and third exponent are the other two roots of this polynomial. 


\section{Equivalence of matrix elements}

So far we mainly studied the statistics of the upper diagonal element $M_{11}$ of the stability matrix $M$. At this point now we can discuss how the results for the cumulant-generating function and the moments can be transferred to the other elements of $M$.

The differential equation (15) for $M_{22}$ can be integrated similarly to the one for $M_{11}$, from which we obtain analogously to Eq. (46) the formal solution

$$
M_{22}\left(t_{0}\right)=1+\sum_{n=1}^{\infty} \prod_{k=1}^{n} \int_{0}^{t_{k-1}} d t_{k}\left(t_{k}-t_{k+1}\right) \frac{v\left(t_{k}\right)}{m} .
$$

Here we defined in each term of order $n$ that $t_{n+1}=0$. It follows by direct computation that the first two moments of $M_{22}$ are identical to those of $M_{11},\left\langle M_{22}\right\rangle=\left\langle M_{11}\right\rangle,\left\langle M_{22}^{2}\right\rangle$ $=\left\langle M_{11}^{2}\right\rangle$. These explicit results already suggest that the statistics of the two diagonal matrix elements is the same. Indeed, the transformation $t_{k}=t-\tilde{t}_{n+1-k}, v(t-\tilde{t})=\tilde{v}(\tilde{t})$ brings Eq. (49) into the form of Eq. (46) and leaves the properties of the Gaussian noise (11) invariant. Hence even the transient behavior of the diagonal elements is completely identical, for arbitrary values of $V_{2}^{\prime}$.

The results for the cumulant-generating function $\eta(\xi)$ (hence also the growth rates of the moments, but not the transient behavior) can also be transferred to the off-diagonal matrix elements of $M$ : The element $M_{12}$ fulfills the same differential equation as $M_{11}$, see Eq. (14), while $M_{21}$ fulfills the same differential equation as $M_{22}$. The initial conditions of the off-diagonal matrix elements differ from those of the diagonal elements. However, according to Eq. (25) this only affects the function $f(\xi)$ in the subleading corrections of the cumulant-generating function [which, for the example of the second moment, results in factors in front of the exponential functions which are different from those in Eq. (48)].

Let us add that from Eqs. (46) and (49), we find for $V_{2}^{\prime}$ $=0$ the cross-correlator $\left\langle M_{11} M_{22}\right\rangle=\frac{1}{2}+\frac{1}{2}\left\langle M_{11}^{2}\right\rangle$. Collecting the results, this gives $\langle\operatorname{tr} M\rangle=2$,

$$
\left\langle(\operatorname{tr} M)^{2}\right\rangle=1+e^{\mu(2) t / t_{c}}+2 \operatorname{Re} e^{-(-1)^{1 / 3} \mu(2) t / t_{c}} .
$$

\section{DISCUSSION}

In this work, we presented a uniform approach to the asymptotic statistics of finite-time Lyapunov exponents, for the model (described in Sec. II) of a particle moving in a random time-dependent potential. The cumulant-generating function $\eta(\xi)$ was found to be directly proportional to the eigenvalue $\mu(\xi)$ of a parametrized differential equation, defined by Eqs. (22). This facilitated an effective analysis of the statistics, including the non-Gaussian deviations of the distribution function. These deviations are especially important for the moments of the elements of the stability matrix, since their growth rate cannot be predicted by the Gaussian approximation Eq. (37).

We limited our attention to the case of time-dependent Hamiltonian systems with a single degree of freedom and a Hamiltonian (5) which is of the special type of kinetic energy plus potential energy, with time dependence only in the potential energy. This case is of particular interest because of its direct applicability to specific dynamical systems as in the random wave-propagation problem of Refs. [22,23], and because of its applicability to one-dimensional wave localization. For the Hamiltonian (5), the matrix $K$ in the differential equation (8) is purely off-diagonal, with fluctuations only in the lower-left element. For Hamiltonians which do not separate into kinetic and potential energy, the differential equation (5) for $M$ involves the matrix $K$ in the more general form

$$
K=\left(\begin{array}{ll}
K_{11} & K_{12} \\
K_{21} & K_{22}
\end{array}\right)=\left(\begin{array}{cc}
\frac{\partial^{2} H}{\partial x \partial p} & \frac{\partial^{2} H}{\partial p^{2}} \\
-\frac{\partial^{2} H}{\partial x^{2}} & -\frac{\partial^{2} H}{\partial x \partial p}
\end{array}\right) .
$$

A generalized statistical model now arises by introducing noise into all of the matrix elements of $K$. (One may also allow for correlations between the different matrix elements or for finite correlation times by introducing auxiliary variables for the noise in the standard way.)

Let us point out two particular cases for which a statistical description promises to result in direct applications to physical situations of interest. One case is more relevant to wavefunction localization while the other is more relevant for chaotic dynamics.

(a) The diagonal elements $K_{11}=-K_{22}=0$ still vanish identically, but both off-diagonal elements $K_{12}$ and $K_{21}$ fluctuate with a vanishing mean. This situation is related to the band-center case of one-dimensional localization in the Anderson model $[41,43,44]$ (where space is discretized on the lattice), since at the band center the effective mass of the particle diverges (and hence the mean of $K_{12}$ vanishes).

(b) Dynamics which are isotropic in phase space, in the sense that the Hamiltonians $H(p, x, t)$ statistically do not single out any specific direction in phase space. In this case one would encounter independent fluctuations of all four matrix elements $K_{i j}$ with identical amplitude and vanishing mean. Hamiltonian dynamics gives rise to the further constraint $K_{11}=-K_{22}$.

It would be interesting to compare the outcome of an analysis of this model with the findings in the literature $[15,16]$, which indicate a certain degree of robustness (if not universality) of the distribution of finite-time Lyapunov exponents in chaotic maps.

\section{ACKNOWLEDGMENTS}

We gratefully acknowledge useful discussions with Igor Gornyi, Philippe Jacquod, and Holger Kantz, and especially with Steven Tomsovic, who motivated us to study this problem.

\section{APPENDIX: INTEGRALS FOR THE HIGHER CUMULANTS}

The cumulants of order $n$ result from the recursion relations Eq. (33) in the form of $2 n$-fold integrals. Usually, the 
numerical evaluation of such integrals for large $n$ is very time consuming, since the number of points on a grid covering the integration domain with lattice constant $(1 / N), N$ $\gg 1$, grows rapidly with $n$ as $N^{2 n}$. However, the integrands in Eq. (33) factorize and the expense of the integration can be reduced from exponential to algebraic $n$ dependence $\sim n N$. The principle can be demonstrated for the example of the twofold integral,

$$
\begin{gathered}
I^{(1)}=\int_{-z_{0}}^{z_{1}} d z I^{(2)}(z), \\
I^{(2)}(z)=g(z) \int_{-z_{0}}^{z} d y I^{(3)}(y),
\end{gathered}
$$

where $g$ is an arbitrary function and $I^{(3)}$ may itself be a multidimensional integral.

We introduce an index $m$ which denotes that the argument of a function is taken at the $m$ th lattice point on the appropriate axis of the grid. The initial values of $I_{m}^{(n)}$ at $m=0$ (the lower integration boundary) are zero. We now can write recursively, by incrementally increasing the integration variables,

$$
\begin{gathered}
I_{m+1}^{(2)}=\frac{g_{m+1}}{g_{m}} I_{m}^{(2)}+\frac{1}{N} g_{m} I_{m}^{(3)}, \\
I_{m+1}^{(1)}=I_{m}^{(1)}+\frac{1}{N} I_{m+1}^{(2)} .
\end{gathered}
$$

Moreover, when $I^{(3)}$ itself is a multidimensional integral of type $I^{(1)}$, its current value can be obtained recursively in the same way as the value of $I^{(1)}$. Since each additional integral will give rise to only one additional equation [similar either to Eq. (A2a) or to Eq. (A2b)], the number of operations grows linearly with $n$, as advertised above. [The recursion relations (A2) have the additional advantage for the present problem that they avoid overflow and underflow in the evaluation of the kernel $K(y, z)=\exp \left(y^{3} / 3+V_{2}^{\prime} y-z^{3} / 3-V_{2}^{\prime} z\right)$.]
[1] E. Ott, Chaos in Dynamical Systems (Cambridge University Press, Cambridge, 1993).

[2] H. Fujisaka, Prog. Theor. Phys. 70, 1264 (1983).

[3] P. Grassberger and I. Procaccia, Physica D 13, 34 (1984).

[4] R. Badii and A. Politi, Phys. Rev. A 35, 1288 (1987).

[5] T. Tél, Phys. Rev. A 36, 2507 (1987).

[6] P. Grassberger, R. Badii, and A. Politi, J. Stat. Phys. 51, 135 (1988).

[7] M. A. Sepúlveda, R. Badii, and E. Pollak, Phys. Rev. Lett. 63, 1226 (1989).

[8] S. Vaienti, J. Stat. Phys. 56, 403 (1989).

[9] F. J. Romeiras, C. Grebogi, and E. Ott, Phys. Rev. A 41, 784 (1990).

[10] A. Crisanti, G. Paladin, and A. Vulpiani, Products of Random Matrices (Springer, Berlin, 1993).

[11] B. Eckhardt and D. Yao, Physica D 65, 100 (1993).

[12] C. Amitrano and R. S. Berry, Phys. Rev. E 47, 3158 (1993).

[13] M. H. Ernst, J. R. Dorfman, R. Nix, and D. Jacobs, Phys. Rev. Lett. 74, 4416 (1995).

[14] A. Adrover and M. Giona, Physica A 253, 143 (1998).

[15] A. Prasad and R. Ramaswamy, Phys. Rev. E 60, 2761 (1999).

[16] F. K. Diakonos, D. Pingel, and P. Schmelcher, Phys. Rev. E 62, 4413 (2000).

[17] H. Yamada and T. Okabe, Phys. Rev. E 63, 026203 (2001).

[18] E. Aurell, G. Boffetta, A. Crisanti, G. Paladin, and A. Vulpiani, J. Phys. A 30, 1 (1997).

[19] I. L. Aleiner and A. I. Larkin, Phys. Rev. B 54, 14423 (1996); Phys. Rev. E 55, R1243 (1997).

[20] O. Agam, I. Aleiner, and A. Larkin, Phys. Rev. Lett. 85, 3153 (2000).

[21] S. Oberholzer, E. V. Sukhorukov, and C. Schönenberger, Nature (London) 415, 765 (2002).

[22] M. A. Wolfson and S. Tomsovic, J. Acoust. Soc. Am. 109, 2693 (2001).

[23] M. G. Brown, J. A. Colosi, S. Tomsovic, A. Virovlyansky, M.
A. Wolfson, and G. M. Zaslavsky, e-print nlin.CD/0109027.

[24] R. A. Jalabert and H. M. Pastawski, Phys. Rev. Lett. 86, 2490 (2001).

[25] Ph. Jacquod, P. G. Silvestrov, and C. W. J. Beenakker, Phys. Rev. E 64, 055203 (2001).

[26] F. M. Cucchietti, H. M. Pastawski, and D. A. Wisniacki, Phys. Rev. E 65, 045206(R) (2002).

[27] N. R. Cerruti and S. Tomsovic, Phys. Rev. Lett. 88, 054103 (2002).

[28] T. Prosen, Phys. Rev. E 65, 036208 (2002).

[29] Ph. Jacquod, I. Adagideli, and C. W. J. Beenakker, e-print nlin.CD/0203052.

[30] F. M. Cucchietti, C. H. Lewenkopf, E. R. Mucciolo, H. M. Pastawski, and R. O. Vallejos, Phys. Rev. E 65, 046209 (2002).

[31] B. V. Chirikov, Phys. Rep. 52, 263 (1979).

[32] P. W. Anderson, Phys. Rev. 109, 1492 (1958).

[33] R. E. Borland, Proc. R. Soc. London, Ser. A 274, 529 (1963).

[34] R. Landauer, Philos. Mag. 21, 863 (1970).

[35] N. F. Mott and W. D. Twose, Adv. Phys. 10, 107 (1961).

[36] For reviews on localization see, e.g., P. A. Lee and T. V. Ramakrishnan, Rev. Mod. Phys. 57, 287 (1985); B. Kramer and A. MacKinnon, Rep. Prog. Phys. 56, 1469 (1993); C. W. J. Beenakker, Rev. Mod. Phys. 69, 731 (1997); A. D. Mirlin, Phys. Rep. 326, 259 (2000).

[37] A. Crisanti, G. Paladin, and A. Vulpiani, Phys. Rev. A 39, 6491 (1989).

[38] H. L. Frisch and S. P. Lloyd, Phys. Rev. 120, 1175 (1960).

[39] B. I. Halperin, Phys. Rev. 139, A104 (1965).

[40] I. M. Lifshitz, S. A. Gredeskul, and L. A. Pastur, Introduction to the Theory of Disordered Systems (Wiley, New York, 1988).

[41] H. Schomerus and M. Titov, e-print cond-mat/0208457.

[42] Two complex-conjugate eigenvalues with the largest real part sometimes exist for odd $\xi$. For simplicity, we will unrestrictedly refer to "the" largest eigenvalue also in these cases, by 
which we then mean either of the two eigenvalues. Moreover, note that no modulus signs appear in Eq. (39). This interpretation of the eigenvalue $\mu$ for odd $\xi$, which can also be motivated from analyticity considerations, is confirmed by the explicit computation of the first moment of $M_{11}$ in Sec. IV B.
[43] M. Kappus and F. Wegner, Z. Phys. B: Condens. Matter 45, 15 (1981).

[44] I. Goldhirsch, S. H. Noskowicz, and Z. Schuss, Phys. Rev. B 49, 14504 (1994). 